Meta

Journal des traducteurs

Translators' Journal

\title{
Traduction en simulation ou en professionnel : le choix du formateur
}

\section{Élisabeth Lavault-Olléon}

Volume 43, numéro 3, septembre 1998

URI : https://id.erudit.org/iderudit/003423ar

DOI : https://doi.org/10.7202/003423ar

Aller au sommaire du numéro

Éditeur(s)

Les Presses de l'Université de Montréal

ISSN

0026-0452 (imprimé)

1492-1421 (numérique)

Découvrir la revue

Citer cet article

Lavault-Olléon, É. (1998). Traduction en simulation ou en professionnel : le choix du formateur. Meta, 43(3), 364-372. https://doi.org/10.7202/003423ar
Résumé de l'article

Formation professionnelle et formation universitaire ne font pas toujours bon ménage, surtout dans le domaine de la traduction qui bénéficie d'une place traditionnelle dans l'université. Ayant choisi ici de traiter de la pratique de la traduction en contexte universitaire, l'auteur de cet article décrit les différentes formules qui permettent de faire évoluer la traduction universitaire dans une optique professionnelle, par des exercices spécifiques, des traductions en simulation et des traductions réellement commanditées et réalisées "en temps réel". L'exemple d'un projet de traduction collective mené à bien dans le cadre de la formation de traducteurs de l'Université Stendhal de Grenoble est proposé comme base de réflexion sur des pratiques pédagogiques qui prépareraient mieux les étudiants à s'insérer rapidement dans le monde professionnel. 


\title{
TRADUCTION EN SIMULATION OU EN PROFESSIONNEL : LE CHOIX DU FORMATEUR
}

\author{
ÉLISABETH LAVAULT \\ Université Stendhal Grenoble-3, Grenoble, France
}

\begin{abstract}
Résumé
Formation professionnelle et formation universitaire ne font pas toujours bon ménage, surtout dans le domaine de la traduction qui bénéficie d'une place traditionnelle dans l'université. Ayant choisi ici de traiter de la pratique de la traduction en contexte universitaire, l'auteur de cet article décrit les différentes formules qui permettent de faire évoluer la traduction universitaire dans une optique professionnelle, par des exercices spécifiques, des traductions en simulation et des traductions réellement commanditées et réalisées «en temps réel». L'exemple d'un projet de traduction collective mené à bien dans le cadre de la formation de traducteurs de l'Université Stendhal de Grenoble est proposé comme base de réflexion sur des pratiques pédagogiques qui prépareraient mieux les étudiants à s'insérer rapidement dans le monde professionnel.
\end{abstract}

\begin{abstract}
University training does not always provide the practical knowledge that professionals require, especially in the field of translation, especially in French universities where a strong academic tradition prevails. This paper considers the role of practice in the training of translators and describes different means of imparting practical skills, including specific exercises, simulated translations and the introduction of direct professional experience into the academic curriculum. A group translation project carried out by the students at Stendhal University is used as a basis for discussion of ways and means of better preparing students to adapt to the professional world.
\end{abstract}

\section{INTRODUCTION}

Les formations de traducteurs se sont multipliées en France ces dernières années, nées d'un besoin d'ouverture de l'université aux études dites «professionnalisantes» et encouragées par un marché demandeur en traduction technique. Elles incluent généralement des cours de méthodologie de la traduction, voire de traductologie, une formation aux outils du traducteur (TAO, outils de gestion de terminologie), et certaines renforcent aussi les savoirs thématiques dans le domaine du droit, de l'édition, des sciences ou de la technologie. Mais c'est la pratique de la traduction qui se taille bien entendu la part du lion, la pratique intensive, multiforme, pluridisciplinaire, et de préférence sous contrainte selon les délais et les exigences des professeurs. Ces enseignants-traducteurs jouent le double rôle de réviseurs-correcteurs et de formateurs-évaluateurs et doivent relever le défi que posent les exigences parfois contradictoires d'une formation professionnelle et d'un contexte universitaire. La pratique de la traduction, exercice universitaire classique, peutelle suffisamment évoluer dans ce contexte pour véritablement former des traducteurs, et dans quelle mesure une formation universitaire est-elle capable de devenir une formation réellement «professionnalisante»?

Meta, XLIII, 3, 1998 


\section{LA TRADITIONNELLE VERSION}

La traduction dans l'université française évoque inéluctablement la version et le thème, ces exercices hérités de l'enseignement du latin qui règnent en maître dans tous les concours nationaux de recrutement des enseignants de langues, en particulier le CAPES et l'agrégation.

Dans différents articles traitant de la différence entre traduction pédagogique et traduction professionnelle, j'ai moi-même dénoncé la place excessive de la version traditionnelle (un extrait court — 150 à 300 mots — le plus souvent tiré d'une œuvre littéraire, parfois d'un article de presse, donné à traduire sans autres éléments contextuels que le nom de l'auteur ou du journal, exercice pratiqué sans aucun dictionnaire ni documents) :

À la lumière de la théorie interprétative, la version dans sa forme traditionnelle semble un non-sens. S'il est vrai que traduire signifie dans un premier temps interpréter pour comprendre, que penser d'un exercice qui défie toutes les lois de la communication, la traduction d'un énoncé isolé, tronqué, coupé de la réalité d'une situation d'énonciation dont l'auteur, le destinataire, le contexte sont délibérément occultés ? D'autre part, quel niveau de traduction peut-on espérer de traducteurs-élèves qui ne possèdent souvent ni la compétence linguistique pour bien comprendre le texte, ni la connaissance des éléments extralinguistiques permettant son interprétation? (Lavault $1987: 120$ et $1993: 127$ )

Je me suis également fait l'écho des constatations des formateurs de traducteurs qui reconnaissent les méfaits de ces exercices artificiels et les handicaps qu'ils créent chez les futurs étudiants-traducteurs :

Les enseignants des écoles de traduction se plaignent d'ailleurs d'avoir à pratiquer un véritable désapprentissage pour éliminer les habitudes prises en cours de langues. Ils font remarquer que les étudiants qui arrivent ont été «rigidifiés par un enseignement qui leur a appris des mots et des équivalences». Ils s'attachent au signifié de chaque mot plutôt qu'au sens de l'énoncé et n'arrivent pas à trouver des formulations spontanées dans leur langue. Il faut alors lutter contre les réflexes de littéralité que des années d'apprentissage leur ont inculqués. (Lavault 1993 : 130)

Ainsi rejetée comme exercice de formation à la traduction professionnelle, la version garde sa justification et ses défenseurs dans le domaine de la stylistique comparée et peut donner lieu à des exercices intéressants visant à approfondir les connaissances linguistiques des étudiants (Ballard 1987). Dans une formation qui se veut professionnelle, elle est difficilement justifiable.

\section{LES ALTERNATIVES}

En ce qui concerne l'apprentissage de la traduction dans le cadre d'une formation de traducteurs professionnels à l'université, plusieurs travaux de types différents peuvent être proposés, que je placerai dans trois catégories :

1) des exercices spécifiques gradués, tirés ou non d'un corpus de traducteurs, et relevant d'une approche didactique de la traduction professionnelle;

2) des traductions en simulation, c'est-à-dire des textes donnés dans leur intégralité, qui ont été initialement traduits en situation réelle par l'enseignant et sont réutilisés en classe;

3) des traductions en professionnel, à faire en temps réel, commanditées par un client et qui font l'objet d'une véritable transaction professionnelle. 
Ces trois catégories de travaux peuvent coexister mais il est vrai qu'elles évoluent souvent avec la formation, la première catégorie cédant peu à peu la place à la deuxième puis à la troisième en fin de cursus.

\section{LES EXERCICES SPÉCIFIQUES}

L'approche de Jean Delisle, qui relève d'une didactique de la traduction, me semble un passage obligé de toute formation de traducteurs. S'appuyant sur la théorie interprétative pour les principes de base, sur l'analyse du discours pour la pratique, Jean Delisle a isolé un certain nombre d'objectifs à acquérir pour les apprentis traducteurs, qui sont autant d'obstacles bien connus de la traduction de l'anglais vers le français. Le travail de traduction proposé porte sur des textes mais aussi sur des listes d'exemples hors-contexte tirés de textes pragmatiques traitant d'économie et de technologie. Ces exemples permettent de repérer les difficultés récurrentes qui sont triées, analysées et traitées sans dogmatisme. Les exercices proposés dans La traduction raisonnée (Delisle 1993) sont sans aucun doute un moyen efficace d'acquérir des réflexes de traducteur tout en perfectionnant sa connaissance des deux langues.

Dans la même optique, la plupart des enseignants-traducteurs ont, dans leurs réserves, un certain nombre de textes ou d'extraits qu'ils utilisent régulièrement pour leur valeur didactique. Le travail collectif de correction d'une mauvaise traduction (un mode d'emploi incompréhensible, par exemple) se révèle plus efficace que tout discours théorique sur la rédaction technique, surtout lorsqu'il est fait en situation, avec un accès à l'appareil dont on décrit le fonctionnement. Chaque enseignant peut ainsi établir une série d'exercices gradués qui familiarisent progressivement l'étudiant avec les difficultés spécifiques du métier. Ces exercices ne sont d'ailleurs pas forcément des exercices de traduction. Il peut s'agir de recherche documentaire rapide sur un sujet pointu, par exemple, ou de réécriture dans la langue cible.

\section{LA TRADUCTION EN SIMULATION}

Le travail que je viens d'évoquer s'impose dans les premières années d'une formation de traducteurs. Il se double d'un travail de traduction guidée sur des corpus de traducteurs regroupant des textes traduits préalablement par l'enseignant-traducteur dans sa pratique professionnelle. Il s'agit de traduction en simulation : l'étudiant ne traduit pas pour un client qui souhaite connaître le sens d'un texte ou le diffuser et qui commandite la traduction, mais pour un enseignant-traducteur qui l'a déjà traduit et le connaît bien, ayant fait toutes les recherches nécessaires pour sa traduction et ayant parfois lui-même subi les interrogations, remarques et corrections d'un réviseur. La traduction en simulation s'effectue donc dans le cadre d'une relation de maître à élève. Rien n'empêche l'enseignant de redonner un texte «qui marche bien» plusieurs fois. Dans ce domaine de la simulation, l'authenticité réside dans le support, à savoir un texte qui a effectivement fait l'objet d'une traduction dans son intégralité, et dans l'expérience vécue et le rôle joué par l'enseignant. Ce dernier peut ajouter des contraintes qui s'approchent de celles qu'il a lui-même subies : délais courts, mise en pages particulière, adjonction d'un glossaire, etc. Il peut aussi bien jouer le rôle d'un donneur d'ordres qui fournit une documentation ou une terminologie que celui d'un donneur d'ordres inaccessible, pour faire réagir les étudiants aux divers cas de figure.

La traduction en simulation offre de nombreux avantages :

- le travail de préparation est diminué pour l'enseignant qui a déjà traduit le texte; 
- si le texte est donné plusieurs années, il se bonifie au fur et à mesure des nouvelles trouvailles des étudiants;

- l'enseignant sait où sont les difficultés et peut prévoir une explication, un travail approfondi sur ces difficultés, travail qui lui aussi se bonifie au cours des années;

- l'enseignant maîtrise tous les éléments : pas de relation-client à gérer (ce qui n'exclut pas d'ailleurs de mentionner les réactions du client initial), pas de délais impossibles, recherche documentaire et terminologique déjà effectuée etc.;

- l'enseignant peut donner les textes à son gré, en établissant une progression qui tient compte des difficultés liées au sujet, de la longueur, des délais.

\section{LA TRADUCTION EN PROFESSIONNEL}

Une formation de traducteurs ne peut cependant pas se limiter à des simulations, et l'introduction dans le cursus de travaux de traduction non simulés, qui répondent à la demande ponctuelle d'un client, semble aller de soi dans une formation professionnelle. Elle se heurte pourtant à de nombreux obstacles pratiques et légaux inhérents à l'organisation d'une activité professionnelle rémunérée dans le cadre de l'université.

\section{Première formule (sans rémunération)}

Elle implique l'obligation pour l'étudiant de réaliser ce qu'on appellera un mémoire de traduction ou une traduction longue, c'est-à-dire une traduction d'au moins dix à quinze pages d'un texte jamais traduit, en général à la pointe de la technique ou de la recherche scientifique, et commandité par un donneur d'ordres. C'est ce qui est demandé, par exemple, pour obtenir la maîtrise de langues étrangères appliquées «Traduction spécialisée» à l'Université Stendhal de Grenoble. Cette formule excluant la rémunération, le commanditaire s'engage, pour bénéficier d'une traduction gratuite, à effectuer une relecture technique commentée du texte traduit de façon à ce que l'étudiant puisse le corriger et livrer un produit fini de qualité. Notons que cette activité ne casse pas le marché, les articles traduits étant généralement fournis par des chercheurs qui n'auraient pas envisagé de payer un traducteur parce qu'ils estimaient «comprendre» suffisamment les textes ou parce qu'ils ne disposaient pas d'un budget suffisant.

Dans ce cas, l'étudiant se trouve dans une situation professionnelle : une commande d'un texte qui n'a jamais été traduit, pour un commanditaire identifié et réel dans des délais précisés. Chaque étudiant se voit attribuer sa traduction et se retrouve donc seul, confronté à un texte spécialisé qu'il ne comprend pas forcément à la première lecture. Il doit se débrouiller sans aucune aide de l'enseignant pour se documenter sur le sujet, trouver des experts, rechercher la terminologie, et rendre une traduction propre dans un délai relativement court pour un étudiant occupé également par d'autres travaux. Il doit ensuite saisir les corrections effectuées par l'enseignant et par le commanditaire.

Pour que ce travail ait le poids d'un mémoire de maîtrise, l'étudiant doit soutenir sa traduction devant toute la classe. Il explique les caractéristiques de son sujet, les démarches effectuées, les difficultés rencontrées, etc., et, pour couronner le tout, il doit «raconter» à ses camarades, en termes simples, le contenu de l'article qu'il a traduit et répondre à leurs questions.

Cette confrontation brutale avec le texte de spécialité est stimulante et déterminante pour le choix de carrière de l'étudiant, chez qui, dans la majorité des cas, se produit un véritable déclic. La soutenance, en particulier, s'avère fructueuse pour tout le groupe grâce à l'analyse des difficultés spécifiques de chaque traduction et à la confrontation des problèmes rencontrés dans des textes relevant de domaines variés. 
L'inconvénient majeur de cette formule est le travail considérable que l'enseignant doit fournir. Il doit, en premier lieu, contacter les chercheurs, trouver suffisamment de textes au bon moment de l'année et les répartir équitablement. Même en admettant que la recherche des textes puisse être assurée par les étudiants qui doivent alors se trouver euxmêmes une commande de traduction, il lui reste néanmoins à veiller à la bonne répartition des travaux et, surtout, à les corriger, ce qui nécessite un interminable travail de recherche et de relecture (en laissant heureusement la relecture technique au demandeur). Il lui faut aussi organiser une soutenance pour chaque traduction et mettre des notes...

Il serait certes bien plus facile de donner le même texte en parallèle à deux étudiants ou à deux groupes, ou encore de réutiliser un texte d'une année sur l'autre, mais tout l'apport professionnel serait faussé, l'opération de traduction ne serait plus authentique et les étudiants perdraient une grande partie de leur motivation.

Malgré tous ses avantages sur la traduction en simulation, cette première formule reste cependant tronquée pour deux raisons : d'abord, elle n'implique pas de négociation financière entre traducteur et commanditaire. Ensuite, les documents traduits reflètent plutôt la demande du campus que celle du marché. C'est une demande en quelque sorte «provoquée» par la formation de traducteurs spécialisés, puisque, sans l'intervention du formateur ou de l'étudiant, les articles en question n'auraient probablement pas fait l'objet d'une traduction.

\section{Deuxième formule}

L'idéal dans une formation de traducteurs est de faire réaliser une traduction en professionnel, «en temps réel», véritablement commanditée et rétribuée par un client. Au travail précédent s'ajoute tout l'apprentissage de la négociation commerciale, c'est-à-dire de la transaction financière et de la gestion de la relation avec le client, avec, au bout du compte, une véritable rémunération pour l'étudiant.

Si idéal qu'elle soit, cette pratique se heurte en France à une difficulté majeure : comment faire rémunérer légalement les étudiants qui ont fait la traduction ? Faute d'avoir résolu cette question, la majorité des formations se cantonne à la traduction en simulation ou à la première formule décrite ci-dessus.

La solution réside dans la création d'une «Jeune entreprise», une association à but non lucratif, dont l'objectif est de favoriser le rapprochement entre les étudiants et les entreprises en proposant des services rémunérés dans le cadre de l'université. Ces associations sont encore assez rares dans le domaine de la traduction et j'aimerais, dans cet article, faire part de l'expérience d'une Jeune entreprise créée dans le cadre du DESS «Traduction spécialisée et production de textes multilingues» de l'Université Stendhal. La Jeune entreprise fonctionne comme suit :

- Les étudiants gèrent l'association et leur clientèle (publicité, accueil téléphonique, secrétariat, comptabilité, etc.) et négocient chaque transaction (commande, devis, facture, etc.). Cela nécessite au départ un peu d'aide d'autres associations du même type et des enseignants, mais très vite les étudiants les plus avancés forment les nouveaux venus. Ces nouvelles tâches peuvent donner lieu à des échanges intéressants sur la gestion des relations commerciales dans le cadre d'un cours de méthodologie, en particulier sur la réalisation d'un cahier des charges.

- L'enseignant n'a plus à gérer les relations avec les clients pour obtenir des traductions à donner aux étudiants, il n'a plus besoin de provoquer la demande auprès de chercheurs.

- La relecture finale peut être assurée par les réviseurs du client (lorsque celui-ci est un éditeur), ce qui soulage considérablement l'enseignant. Si la traduction doit être 
de qualité «diffusable» (pour ce concept, voir Gouadec 1990 : 55), un enseignant peut choisir d'en assurer la révision en se faisant rétribuer.

- Les étudiants deviennent vite très autonomes et s'organisent pour suivre la demande du marché : les délais ne sont plus des délais scolaires mais les habituels cassetête des traducteurs qui nécessitent de travailler tout le week-end par exemple. Comme ils sont rétribués, les étudiants assument ces charges supplémentaires de bon cœur...

- Enfin, les textes proposés correspondent davantage au marché : ce sont plutôt des livres, soit spécialisés soit grand public, des brochures, des documents technicocommerciaux ou industriels.

La Jeune entreprise semble la solution rêvée pour une formation professionnalisante, mais son insertion dans le cursus universitaire proprement dit n'est pas facile et un certain nombre de questions se posent :

Quelle est alors la place de l'enseignant? Se doit-il de donner des notes pour ce type de travaux, et comment les évaluer ? Est-il en mesure de contrôler tous les travaux effectués par la Jeune entreprise ? Faut-il considérer les travaux effectués pour les clients de la Jeune entreprise comme des travaux annexes, exempts d'évaluation (la rémunération et l'acquisition d'une expérience authentique fournissant la motivation pour les étudiants, le respect du contrat et la rémunération étant une forme d'évaluation) ? Dans ce cas, faut-il dissocier les travaux de la Jeune entreprise de la formation et réserver l'évaluation à des travaux en temps limité et en salle? Mais alors, comment respecter l'équilibre entre les tâches proposées et évaluées par les enseignants et les travaux rémunérés qui motivent davantage les étudiants et leur prennent beaucoup de temps ? Enfin, comment assurer la qualité des travaux de la Jeune entreprise et faire en sorte qu'elle soit, par son efficacité et son rayonnement, un véritable tremplin pour l'insertion professionnelle des jeunes diplômés ?

On trouvera quelques éléments de réponse dans l'étude de cas qui suit.

\section{ÉTUDE DE CAS}

Lorsqu'un grand éditeur parisien a proposé à notre Jeune entreprise la traduction d'un ouvrage d'informatique destiné au grand public (350 pages sur un sujet technique à la mode, à traduire en huit semaines, en plein deuxième trimestre, rétribué au tarif en vigueur), l'offre ne pouvait être refusée. Il était clair, cependant, que la réalisation d'une traduction collective, dans des conditions professionnelles réelles, ne pouvait s'improviser. L'expérience en gestion de projet manquait cruellement, mais le groupe de 14 étudiants, encadré par deux enseignantes-traductrices (une francophone, une anglophone) et un informaticien, a réussi son pari : la traduction a été livrée le jour J, le livre est sorti un mois plus tard et le contrat a été honoré. Les péripéties ont été multiples et sont révélatrices du fossé qui sépare les habitudes de travail en contexte universitaire du travail en professionnel.

\section{Organisation}

Les étudiants ont été divisés en sept groupes qui se sont réparti la traduction ellemême (le livre a été grosso modo découpé en 14 parties sensiblement égales) ainsi que toutes les tâches annexes dont la liste - incomplète à l'usage - avait été réalisée au départ : contacts avec l'éditeur, recherche terminologique et établissement de listes, capture d'écrans sur Internet, mise en pages et application de la feuille de style, indexation, adaptation des légendes, relecture pour le français et l'orthographe, relecture pour la cohé- 
rence terminologique et typographique, homogénéité des menus de chapitres et de parties, etc.

Les enseignants responsables ont assuré la correction de l'intégralité de la traduction. Il semblait indispensable de veiller à la qualité, car il s'agissait du premier contrat de cette envergure. De plus, la traduction étant intégrée dans le cursus comme «traduction longue», il fallait bien donner une note à chaque étudiant.

Le travail des enseignants était donc considérable, mais avec une petite soupape de sécurité, puisque la qualité obtenue, une fois les corrections de l'enseignant saisies, restait «révisable», la traduction devant ensuite être passée au crible du réviseur de la maison d'édition.

\section{Difficultés}

Les difficultés ont été de trois ordres :

\section{Les difficultés techniques}

Ce sont les difficultés techniques inhérentes à ce type de traduction. L'ouvrage décrivait l'utilisation d'une nouvelle version d'un logiciel qui venait de sortir aux ÉtatsUnis et dont la localisation française n'était pas terminée au moment de la traduction, d'où de grandes difficultés pour trouver la documentation et de nombreuses incertitudes sur la terminologie (supputée identique à la version précédente, mais ce n'était pas toujours le cas). Il a fallu rechercher régulièrement sur Internet la version bêta, qui est arrivée partiellement traduite, et manipuler le logiciel dans tous les sens pour trouver la traduction de toutes les commandes, de tous les messages d'aide, bref de tous les écrans. L'ouvrage comportait en outre un grand nombre d'illustrations représentant des écrans Internet sous Netscape. Les recherches sur Internet pour retrouver ces écrans avec le logiciel en français ont pris des heures, sans parler de la capture des écrans, manipulation jusqu'à présent absente du cours d'informatique. Enfin, de nombreuses applications assistantes, décrites dans le livre, n'étaient pas disponibles, et de nombreux écrans sont restés introuvables. Ces questions ont finalement été réglées par l'éditeur, mais elles ont beaucoup contrarié les étudiants-traducteurs, pour la première fois confrontés à des problèmes qui dépassaient le cadre universitaire familier et qu'ils étaient incapables de résoudre sans faire intervenir une négociation avec l'éditeur. En contrepartie, il faut reconnaître que traduire un livre sur un sujet qui fait la une de toute la presse (Internet, le Web et ses navigateurs) était particulièrement motivant !

\section{Les difficultés d'organisation}

Elles ont été essentiellement dues à un manque d'expérience en gestion de projet. Travailler à 14 nécessite une gestion parfaite de la communication et de la transmission des données. Les réunions où chacun expose ses difficultés à son gré, où des conseils, des consignes, des contrordres sont donnés oralement dans le brouhaha et la fatigue ont donné des résultats catastrophiques : consignes de terminologie ou de mise en pages mal appliquées, par exemple, et traduction à recorriger entièrement, etc. Un système d'affichage et de messagerie électronique bien pensé est indispensable et une personne compétente doit en être responsable. Tous les rôles doivent impérativement être définis au départ et les personnes impliquées doivent être facilement «joignables».

Indépendamment des problèmes techniques spécifiques au type d'ouvrage traduit, la formation des étudiants en informatique s'est révélée insuffisante, surtout en ce qui concerne la gestion des postes de travail et des fichiers. Un exemple : l'homogénéisation des noms de fichiers n'a pas été faite au départ («chap 1» ou «chapitre 1» ou «Premier chapitre», etc.), d'où des difficultés pour regrouper en bon ordre tous les fichiers pour la livrai- 
son. L'utilisation d'une feuille de style n'etait pas maitrisée, d'où certaines catastrophes dans les saisies de tableaux.

Si motivés et impliqués qu'ils soient, les étudiants font encore beaucoup d'erreurs par distraction ou inexpérience : corrections mal saisies, sauvegardes oubliées, consignes terminologiques mal formulées et mal appliquées, etc. Les enseignants ont eu tendance à surestimer le degré d'autonomie des étudiants, si bien que le travail d'encadrement et de révision finale (juste avant livraison) a largement débordé les prévisions.

La gestion du temps a été déficiente : trop peu de temps avait été prévu pour la capture d'écrans et la révision, et la traduction s'est terminée dans l'affolement général.

\section{Les difficultés de traduction}

Finalement, la traduction elle-même a occupé une place restreinte dans la gestion totale du projet par rapport à tout le travail de préparation et de relecture. Les étudiants étaient déjà bien formés et beaucoup traduisaient déjà correctement. Bien sûr, il y a eu des fautes, mais il est intéressant de noter que les fautes de sens portaient le plus souvent sur les aspects les moins techniques du texte, ceux où se trouvaient les références à la culture ou à la vie quotidienne. Cependant, le plus frappant, lorsque l'on corrige un texte long traduit par 14 individus différents, c'est l'importance de la phraséologie et du style, même dans un ouvrage technique de ce type. Or le travail de préparation phraséologique avait été relativement négligé au départ (à part pour quelques expressions manifestement récurrentes comme double-click on, right-click on, etc., pour lesquelles un terme avait été choisi au départ, avec l'accord de l'éditeur). En fait, beaucoup d'expressions faisaient l'objet de choix éditoriaux dont on ne s'est préoccupé qu'a posteriori (par exemple «Internet» plutôt que «l'Internet», «voir Chapitre 6» plutôt que «cf. chap 6»...) et qui ont nécessité d'interminables corrections. Le travail terminologique préparatoire avait consisté en un inventaire des termes techniques. Or beaucoup de fautes de traduction ont porté sur des termes simples récurrents qui n'avaient pas été recensés comme techniques et qui auraient dû se trouver dans l'inventaire phraséologique : par exemple, search a database («faire des recherches dans une base de données», et non pas «rechercher une base de données»...), check this site («consulter ce site», «aller voir ce site», et non pas «vérifier»). Enfin, une réflexion sur le style aurait dû être conduite dès la première lecture du livre à traduire, en particulier pour toutes les tournures récurrentes comportant des modaux ou des propositions avec if, caractéristiques de ce genre d'ouvrage. Certains étudiants ont calqué les tournures américaines, alors que d'autres ont d'emblée allégé le style en employant l'impératif en français, d'où une hétérogénéité marquée d'un chapitre à l'autre qui a fait s'arracher les cheveux au correcteur.

\section{Bilan}

Le découpage du livre en 14 parties a été choisi uniquement parce qu'il fallait noter les étudiants. Malheureusement, la note qui a été donnée reflète les qualités de chaque étudiant en traduction, mais prend peu en compte la façon dont chaque groupe s'est acquitté des autres tâches, toutes si différentes et donc difficiles à évaluer. Certains étudiants, dont la traduction était plutôt médiocre, se sont démenés comme des diables pour réaliser des captures d'écran difficiles, ou ont passé des heures à tester le logiciel à la recherche de la terminologie. D'autres, qui ont bien traduit, se sont peu impliqués dans le travail collectif. Le problème de l'évaluation de travaux professionnels qui couvrent nécessairement beaucoup plus de compétences que la seule compétence de traduction reste donc posé.

Sans cette contrainte d'évaluation, nous aurions procédé à un autre découpage des tâches, en réservant la traduction et la relecture aux meilleurs traducteurs-rédacteurs, les tâches d'organisation et de communication aux plus efficaces et les tâches informatiques 
aux plus dégourdis sur les machines. L'homogénéité de la traduction aurait été meilleure et la tâche du réviseur allégée. Mais il faut bien aussi que les étudiants-traducteurs apprennent à réaliser toutes les opérations qu'implique un projet. La répartition des tâches dans un projet de traduction est donc le deuxième problème, d'autant plus critique qu'en général, tous les étudiants veulent traduire.

L'assurance-qualité reste, à mon avis, le troisième problème. Dans ce cas précis, nous étions soulagés de savoir qu'un réviseur en assurait la responsabilité, mais ce n'est pas toujours le cas. Les enseignants étant généralement débordés, dans quelle mesure peut-on confier la révision aux étudiants ? Faut-il faire appel à des réviseurs extérieurs ? Une des réponses est la négociation préalable et précise de la qualité souhaitée par le client. L'utilisation d'un cahier des charges complet et rigoureux fait partie de la prise en charge du projet et une réflexion collective sur ce point est indispensable (Gouadec 1990 : $55)$.

Enfin, pour que les travaux en professionnel soient bien intégrés dans la formation, il faut qu'ils arrivent à point nommé et que les délais soient raisonnables : les commandes idéales arrivent au plus tôt en décembre, au plus tard fin février, (ce qui permet de bien mettre les étudiants en route avant de se lancer dans les «vrais» travaux), et accordent plusieurs semaines de délai. Mais le marché, comme chacun sait, a d'autres contraintes.

\section{CONCLUSION}

Malgré toutes les difficultés rencontrées, ce projet de traduction a été jugé très positivement par les étudiants qui ont vraiment eu l'impression de «passer professionnels» à cette occasion. Une fois le premier pas franchi, la réalisation de projets de traduction de ce type devient plus facile, surtout si certains étudiants aguerris peuvent apporter leur expérience aux nouveaux venus. Une des solutions aux problèmes d'encadrement et d'organisation réside dans une structure verticale de gestion de projet impliquant des étudiants de plusieurs niveaux. En décrivant cette expérience, j'ai souhaité montrer que les travaux en professionnel sont, dans une formation universitaire, non seulement souhaitables mais réalisables et crédibles. Il est néanmoins évident que leur réussite est conditionnée par l'engagement enthousiaste de l'enseignant responsable et, idéalement, par celui de l'équipe enseignante au complet.

\section{RÉFÉRENCES}

BALLARD, Michel (1987) : La traduction : de l'anglais au français, Paris, Nathan Université

DELISLE, Jean (1980): L'analyse du discours comme méthode de traduction: initiation à la traduction française de textes pragmatiques anglais, Éditions de l'Université d'Ottawa.

DELISLE, Jean (1993) : La traduction raisonnée, Les Presses de l'Université d'Ottawa

GOUADEC, Daniel (1990) : Le traducteur, la traduction et l'entreprise, Paris, Afnor gestion.

LAVAULT, Élisabeth (1987) : «Traduction pédagogique ou pédagogie de la traduction», Le français dans le monde, numéro spécial : Retour à la traduction, Paris, septembre 1987.

LAVAULT, Élisabeth (1993) : «Traduire en LEA, traduire pour communiquer», M. Ballard (dir.), La traduction à l'université, Recherches et propositions didactiques, Presses Universitaires de Lille. 